Videm

\title{
ANALISI COMPARATIVA DEI NOMI DELLA GERARCHIA ECCLESIASTICA IN SLOVENO E CROATO
}

L'argomento di questa ricerca trae spunto da due miei precedenti lavori trattanti l'uno i nomi per la gerarchia ecclesiastica in sloveno, l'altro quelli per il medesimo settore lessicale in croato. Il primo apparve in veste monografica (La Chiesa in Slovena. Analisi filologico-etimologica della gerarchia ecclesiastica con particolare riguardo ai testi del Cinquecento, Centro studi storico-religiosi Friuli-Venezia Giulia, 14, Trieste 1984), jl secondo in forma di articolo nella Rivista di Scienza religiose Studia Patavina (I nomi della gerarchia ecclesiastica in croato, Studia Patavina 37 (1990) 1, Padova 1990, p. 97-117.

Per entrambi gli studi mi è servito da traccia sia per l'apparato bibliografico che per la disposizione della materia trattata il volume del Tagliavini Storia di parole pagane e cristiane attraverso i tempi, Brescia 1963. Il materiale lessicale è stato disposto cosi nei singoli capitoli ovvero paragrafi incominciando dai termini che indicano la Chiesa e genericamente il clero, e proseguendo poi con ordine attraverso la designazione dei vari gradi della gerarchia ecclesiastica: il diacono, il cappellano, il sacerdote, il parroco, il canonico, il vescovo, il cardinale, il papa ed infine i monaci ed i monasteri. La ricerca è stata svolta in entrambi i casi da un punto di vista diacronico iniziando dai primi documenti per risalire alla situazione linguistica odierna attraverso tutti gli strumenti linguistici più importanti quali dizionari, edizioni dei documenti antichi più importanti, edizioni del materiale epigrafico, nonchè il materiale inedito per lo SLA ( = Slovenski lingivistični atlas) e per il dizionario dell'epoca protestante (concessimi entrambi in visione dalla SAZU) e quello per il dizionario dello slavo ecclesiastico di redazione croata, messomi a disposizione dallo Staroslavenski zavod "Svetozar Ritig" di Zagabria.

Il raffronto del materiale lessicale analizzato pone subito in evidenza una notevole quantità di punti in comune, ma anche di differenze ed addirittura di reciproche influenze (anche se non molto frequenti) soprattutto del croato sullo sloveno. Questi rapporti di differenze e similitudini e di reciproche interdipendenze sono dovute a vari fattori: innanzitutto ai diversi centri di irradiazione del cristianesimo in territorio etnico sloveno e croato, al sostrato linguistico al momento della cristianizzazione, ai contatti linguistici con i popoli limitrofi anche in epoche successive alla cristianizzazione nonchè alla stessa confinanza geografica dei due popoli in questione. 
Posiamo asserire genericamente senza soffermarci sui dettagli lessicali (per $\mathbf{i}$ quali rimandiamo alle conclusioni dell'articolo) che si evidenzia subito sia per lo sloveno che per il croato una grande quantità di prestiti dal latino, soprattutto di voci dotte. Per il croato sono da collocare numericamente al secondo posto i prestiti dal greco (mediati soprattutto dallo slavo ecclesiastico e quindi molto numerosi nei documenti glagolitici), mentre per lo sloveno questo tipo di prestiti può dirsi quasi nullo. Molto forte si rivela per lo sloveno l'influsso del germanico che è assai meno evidente nel croato, mentre per ciò che riguarda gli italianismi ed i romanismi questi si collocano addirittura al terzo posto nel croato (frequentissimi in territorio dalmato), mentre nello sloveno sono relegati unicamente a rari prestiti dal friulano. I prestiti dall'ungherese si rivelano un po' più numerosi in croato rispetto allo sloveno. Per entrambe le lingue notiamo inoltre una notevole preponderanza di prestiti rispetto ai calchi.

Se l'influsso del latino ecclesiastico era molto forte, ciò non deve meravigliare, perchè era la lingua ufficiale della Chiesa occidentale. Il latino era altrettanto vivo nella diocesi di Salisburgo come nel patriarcato di Aquileia, i due centri missionari che cristianizzarono gli sloveni, e dominava altresì a Salona e Sirmio, le città dopo Aquileia più importanti per la cristianizzazione dei croati da parte della Chiesa di Roma. Ma i croati si trovarono all'incrocio di due mondi, poichè in parte cristianizzati anche dalla Chiesa orientale di Bisanzio, per cui l'influsso del greco è notevole, mentre per lo sloveno non ha praticamente ragione di esistere. Nello stesso modo si spiega la maggior frequenza del germanico in sloveno rispetto al croato, data l'origine germanica dei missionari, che venivano da Salisburgo. Così il romanzo prevale nella fascia costiera della Croazia per la sua natura mistilingue, mentre questo fenomeno naturalmente non si registra in territorio sloveno. I prestiti dall'ungherese sia in sloveno che in croato sono dovuti a ragioni di carattere soprattutto geografico.

Inoltre la prevalenza dei prestiti sui calchi non deve stupire, anche se nel latino ecclesiastico i calchi linguistici sono solitamente molto numerosi, come del resto in tutte le lingue speciali di carattere più o meno tecnico. Sarà infatti doveroso precisare che anche presso $i$ primi autori cristiani in latino i prestiti erano assai più numerosi rispetto ai calchi, si pensi p.es. in latino a termini comunissimi quali angelus edd

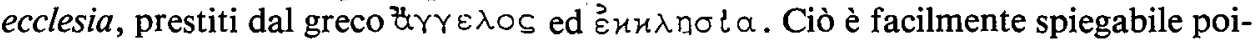
chè il calco, la traduzione cioè con mezzi linguistici propri di un termine designante un concetto del tutto nuovo ad una determinata lingua e cultura, presuppone già una certa evoluzione linguistica. Se si osservano quindi dei prestiti cosi numerosi in una lingua evoluta come era il latino agli inizi del Cristianesimo, non ci si deve stupire dello stesso fenomeno linguistico nello sloveno e nel croato, in special modo per le epoche più antiche.

Tra le prime voci trattate, quali chiesa, clero e chierico, diacono, ecc. si notano più similitudini che differenze, soprattutto perchè trattasi di voci dotte. Così lo slo- 
veno cérkev ed il croato $c \hat{r} k v a^{1}$ appaiono già nel primo documento della letteratura slovena ovverossia croata, cioè nei Brižinski spomeniki e nella Baščanska ploča. Diversa sembra però essere la loro origine. L'etimo delle consimili voci slave è comunque molto problematico ed è stato oggetto di varie discussioni. Data la molteplicità delle forme slavo-ecclesiastiche si sono proposte in linea generale due forme di irradiazione, una germanica (e.gr. aat. kirihha oppure una forma pregotica* kyrikō) ed una greca, un aggettivo sostantivato $x \cup \rho\llcorner a x \delta s,-\hbar,-\delta \vee$ (sott. oixos, $\delta \tilde{\omega} \mu \alpha$ ecc. $)^{2}$.

Per quanto riguarda lo sloveno, data la forma $u$ circuvach attestata nei Brižinski spomeniki secondo il Moszyński ${ }^{3}$ essa deriverebbe dall' a.bav. kirkô, mentre per il croato lo Skok propende, non senza difficoltà, per una diretta derivazione dal greco ${ }^{4}$.

Per designare il clero ed il chierico sia lo sloveno che il croato usano gli stessi termini klêr, klêrik ovverossia klêr, klêrik, prestiti dal latino ecclesiastico clerus e clericus. Le voci slovene compaiono molto tardi, appena nel dizionario del Pleteršnik alla fine del secolo scorso, mentre quelle croate sono databili al XV e rispettivamente XVII secolo ${ }^{5}$. Sotto l'influsso dei testi slavoecclesiastici sono inoltre da citare le forme del croato antico klir (dal XV sec.) e klîrik (dal XIV) ${ }^{6}$ con la conservazione del passaggio $\operatorname{di\eta }>i$.

Per ciò che concerne le denominazioni per il diacono molto semplice si presenta lo slov. diákon, prestito dal lat. eccl. diaconus. Si tratta comunque di un termine che comincia ad essere usato abbastanza tardi, nei dizionari dal Gutsmann in poi, cioè alla fine del Settecento. In epoca precedente è registrato una sola volta nella Bibbia di Dalmatin come postilla a chiarificazione di un passo degli Atti degli Apostoli (IV, $3)^{7}$.

Nell'antico croato la voce è frequente sia nella forma dijakon (fino al XVIII sec.), anche djâkon (dal XVI sec. in poi) e dijak, djâk (quest'ultima dal XV sec. in poi), rispecchianti lo slavo ecclesiastico dijakonŭ e dijakŭ, prestiti dal gr.

In questo articolo gli accenti usati per lo sloveno sono tratti fino ai termini inizianti con la lettera $\breve{s}$ dallo SSKJ, mentre i restanti in mancanza dell'ultimo volume del suddetto dizionario sono presi dallo SP. Gli accenti invece usati per il croato sono basati sull'ARj. Nel caso si riscontrasse un accento non corrispondente ad alcuna delle due opere citate, ciò significa che si riporta l'accento indicato da un determinato autore.

1 Per quanto riguarda le molte varianti čakave e kajkave del termine, riportate fra l'altro da FERLUGA-PETRONIO 1990, si cf. HRASTE-ŠIMUNOVIĆ I, 104 e FINKA I, 2, 257, 262 e 263.

2 La più completa letteratura per quanto concerne questo etimo appare nel KIPARSKY, 247 e nell'articolo di MOSZYNSKI, Najstarsze zasiegi stowiańskich form obocznych * cŕky/*cirüky in Nahtigalov zbornik, Ljubljana 1977, $281-292$.

3 Cf. op. cit., 284.

4 Cf. SKOK I, 275 e RESI VII, 182-185.

5 Cf. $A R j$ s.v. e Mat. Diz. sl. eccl. AI XIV sec. è đatabile la forma più rara klèrig (ibidem).

6 Cf. $A R j$ s.v.

7 Il dato è tratto da Mat. Diz. prot. 
$\delta \iota d u o v o s$ e dal greco tardo $\delta \iota d u o s$. Dal XVIII secolo in poi si riscontrano le

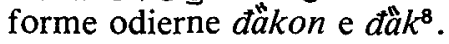

Oltre alle voci dotte dijakon e đăkon, che traggono origine a seconda dei documenti in cui compaiono o dal latino o dal greco, in quest'ultimo caso attraverso la mediazione dello slavo ecclesiastico, soprattutto dei documenti glagolitici, sarà da registrare un altro termine, žakan, circoscritto al territorio del Quarnaro, dell'Istria e di Zara, e documentato dalle epigrafi e dalla letteratura glagolitica. Circa l'etimo di quest'ultimo termine si pensa, soprattutto per il passaggio fonetico $d i \grave{\alpha}>\check{z}$ ad un intermediario romanzo, forse al veneziano zago ${ }^{9}$.

Abbiamo fin qui notato una grande similitudine di termini e significati, le differenze si notano quasi unicamente nelle mediazioni etimologiche del termine. Il quadro lessicale diventa alquanto più composito e vario per le denominazioni che riguardano il cappellano, ma ancor più per il sacerdote ed il parroco. Si tratta in questo caso non soltanto di voci dotte, ma di termini che vengono quotidianamente usati dal popolo e che subirono nell'arco di un millennio, dai primi documenti scritti fino ai nostri giorni, notevoli mutamenti.

Cosi per la denominazione del cappellano notiamo una differenza semantica fra sloveno e croato (eccezion fatta per il kajkavo). Lo sloveno kaplán che incomincia a venir registrato nei dizionari dal Megiser, cioè dal XVII sec., indica il coadiutore del parroco ${ }^{10}$. E' questo un prestito dal mat. kaplān il cui significato primario di sacerdote di una comunità (negli ospedali, nell'esercito, ecc.) si allarga appunto anche a "coadiutore parrocchiale"11.

Il croato invece conosce due termini distinti per queste due funzioni ecclesiastiche, pur restando relegato un termine al contesto dialettale. Il primo, kapèlan, attestato fin dai tempi più antichi (XIV sec.) e derivante dal lat. eccl. capellanus, indica la guida spirituale di una comunità, kaplan invece nel dialetto kajkavo significa unicamente "vice-parroco"12. Si nota in quest'ultimo caso l'influenza dello sloveno sul croato, dovuto alla vicinanza geografica.

Molto vario e interessante è il paragone fra i termini sloveni e croati che indicano il sacerdote. Nello sloveno la voce più diffusa per designare il sacerdote è duhóvnik. Incomincia ad essere attestata tardi, dal dizionario del Pohlin in poi, designando in principio semplicemente il religioso. E' destinata quindi a soppiantare il diffusissimo fár che assunse in epoca protestante una connotazione negativa nei confronti dei sacerdoti cattolici. Era conosciuta però già in epoche precedenti in forma di aggettivo sostantivato duhóven, duhóvni (Krelj, Dalmatin). La sua diffusione era comunque scarsa. L'espressione duhóvni ôca "padre spirituale" risale comunque al Manoscritto di Stična (Stiški rokopis) della prima metà del XV sec. ${ }^{13}$

8 Cf. ARj s.v. đäkon e đâk, MIKL., Lex., 162 e Sl. j. stsl. 1, 484-485.

9 Si cf. per ciò che riguarda le voci in questione SKOK III, 670 e per le attestazioni delle epigrafi e della letteratura glagolitica FUČIĆ, 408 e Mat. Diz. sl. eccl.

10 SSKJ II, 532.

11 Cf. STRIEDTER-TEMPS, 145 e KLUGE-MITZKA, 349.

12 Cf. SKOK II, 41.

13 Cf. GRAFENAUER, St. rkp., 316. 
Il croato dùhovnik a sua volta indica unicamente la guida spirituale, il confessore di una comunità ${ }^{14}$, mentre il termine più diffuso per indicare il sacerdote è svèćenik, voce panslava (sl. eccl. svęštenikŭ ${ }^{16}$ che in sloveno (svečeník) designa unicamente il sacerdote pagano. Nei testi glagolitici è registrata anche la forma sveštenik che indica l'influsso dello slavo ecclesiastico ${ }^{17}$.

Il termine sloveno più antico fár $(<\text { mat. bav. pharr })^{18}$ era diffusissimo fra gli scrittori protestanti del Cinquecento e veniva all'inizio usato indistintamente sia per il sacerdote cattolico che protestante, ma nell'infuocata polemica contro la Chiesa cattolica cominciò ad assumere un significato sempre più dispregiativo che è rimasto immutato fino ai nostri giorni ${ }^{19}$. Per il pastore protestante si adottò in seguito sempre all'epoca della Riforma il termine prídigar "predicatore" $(<$ mat. predigœere, bredigœre ${ }^{20}$. A titolo di curiosità sarà da citare in territorio dalmato, dove si usa tuttora dai tempi più antichi, l'analogo termine, predikator, predikatur, pridikatur per indicare genericamente il predicatore. Esso trae origine o dal lat. praedicator o dall'it. predicatore ${ }^{21}$.

A differenza dello sloveno, per il croato la Riforma protestante non ha inciso in modo determinante sul lessico ecclesiastico né sulla cultura letteraria in generale. Mentre con il protestantesimo gli sloveni si affacciano alla storia letteraria e cominciano a forgiare ed affinare la loro espressione linguistica anche e soprattutto nel lessico religioso, poichè la loro è principalmente in quel periodo letteratura di carattere religioso, la terminologia cristiana in croato si è in buona parte creata già prima, durante i secoli di tradizione glagolitica.

Registriamo così in croato dei termini che sono caduti completamente in disuso nella lingua odierna e che non sono riscontrabili in sloveno, oppure che appartengono in croato esclusivamente alla lingua liturgica, mentre in sloveno possono essere etichettati come dei curiosi relitti lessicali. Ci riferiamo in particolar modo a due termini anticocroati: svètitelj e jerej. Il primo, attestato già nell'antico slavo ecclesiastico e relegato in croato unicamente ai testi glagolitici di carattere liturgico designa di solito i gradi più alti della gerarchia ecclesiastica o personaggi di grande spicco nella Chiesa slava, come ad es. i Santi Cirillo e Metodio ${ }^{22}$. Il secondo, jerej, che è pure ampiamente documentato nei testi dell' antico slavo ecclasiastico, da dove si è diffu-

14 Per il significato di dùhovnik cf. $A R j$ s.v. e SKOK I, 373.

15 Anche in tutte le altre lingue slave sia degli Slavi dell'area cattolica che in quelli dell'area ortodossa le espressioni simili allo sloveno odierno duhóvnik ed agli obsoleti duhóvni e duhóvni ốa indicano esclusivamente il confidente spirituale di una comunità. Per il significato delle voci slave in questione si cf. FERLUGA-PETRONIO, 1984, 53.

16 Cf. Sl. j. stsl. IV, 46.

17 Cf. Mat. Diz. sl. eccl.

18 Cf. STRIEDTER-TEMPS, 115 e BEZLAJ I, 127-128.

19 Si veda a questo proposito PLETERSNIK I, 198 e SSKJ I, 616.

20 Cf. STRIEDTER-TEMPS, 201 e KLUGE-MITZKA, 563.

21 Cf. SKOK III, 40 e SETKA II, 142. Il testo è attestato già nei breviari glagolitici del XIV sec. nelle forme predikatori e pridikaturi (Mat. Diz. sl. eccl.)

22 Cf. ARj s.v., Sl. j. stsl. I, 849 e Mat. Diz. sl. eccl. Per quanto riguarda il particolare significato di "confessore" che svètitel $j$ assume in un caso particolare nell'antico croato si rimanda a FERLUGAPETRONIO 1990, 105. 
so quale designazione ufficiale per indicare il sacerdote in tutte le lingue degli slavi di confessione ortodossa $a^{23}$, è un grecismo (cf. gr. i $€ \rho \in u ́$ s) che è entrato nel croato attraverso la mediazione dei testi glagolitici, e viene usato soltanto nei casi in cui ci si riferisce espressamente alla Chiesa ortodossa, mentre i serbi lo usano tuttora nella lingua liturgica ${ }^{24}$.

In sloveno questa voce appare attestata due volte nella Postila slovenska di Sebastjan $\mathrm{Krelj}^{25}$ per designare la persona di Cristo stesso. Si tratta di un particolare stilistico dovuto esclusivamente alla profonda erudizione di Krelj. A livello dialettale è riscontrabile nella Val di Resia nelle forme iệro ed êrro ${ }^{26}$. Si tratta di un relitto lessicale che finora si presta difficilmente ad una valida spiegazione ${ }^{27}$, dovuto alla particolare posizione geografica dei Resiani.

Sempre nell'ambito dialettale, ma nella regione del Prekmurje riscontriamo in sloveno un altro termine di diffusione panslava, $p o ̈ p^{27}$, nel significato piuttosto singolare di pastore protestante ${ }^{28}$. In questa regione vive infatti a tutt'oggi una comunità protestante. Nello sloveno letterario questo termine designa invece il prete ortodosso.

Il croato $p \delta \bar{p}$ ebbe invece una diffusione assai più ampia. Si trova attestato già dal XIII secolo. Anticamente era - dal punto di vista semantico - l'equivalente di svèćenik, svèštenik oppure di žüpnik e pàroh (termini che designano il parroco). Quindi a somiglianza del tedesco $P f a f f(e)$ cominciò ad essere usato in senso spregiativo tranne che in Istria ed in Dalmazia dove indicava i sacerdoti che celebravano messa in paleoslavo. Nei testi glagolitici infatti il termine è ampiamente usato. Dal XVIII secolo in poi invece questo particolare tipo di sacerdote legato alla tradizione paleoslava comincia ad essere designato con il termine glagòljas ${ }^{29}$.

Sia in sloveno che in croato viene usato nel linguaggio strettamente liturgico prézbiter, rispettivamente, prèzbiter, per indicare genericamente il religioso che è stato consacrato sacerdote, ma soprattutto il superiore in una comunità religiosa. Entrambe le voci traggono origine dal lat. eccl. presbyter, a sua volta grecismo da $\pi \rho \varepsilon \sigma \beta U \tau \varepsilon \rho \circ \varsigma$, che al tempo di Tertulliano viene già usato nel significato di "ministro del culto", inferiore all'episcopus, ma superiore al diaconus. Nell'antico croato riscontriamo però anche le forme prezviter, prozviter, dovute all'influsso

23 Per le corrispondenze nelle lingue slave si cf. FERLUGA-PETRONIO 1984, 57.

24 Cf. Mat. Diz. sl. eccl. e ŠETKA 1 1, 116-117.

25 Informazione desunta da Mat. Diz. prot.

26 Informazione tratta da Mat. SLA.

27 Non tutti gli studiosi sono d'accordo sull'origine dello sl. eccl. popŭ, comunque la maggior parte propende per un prestito dall'aat. pfaffo. Si tratterebbe di un termine introdotto in territorio moravo dai missionari di Regensburg e Salisburgo ed in seguito recepito dagli apostoli Cirillo e Metodio (cf. BRÜCKNER, 430 e KIPARSKY, 260).

28 Cf. Mat. SLA. Sarà interessante notare come anche nel sorabo superiore questo termine indichi il pastore protestante e viene usato dai cattolici proprio per distinguerlo dal proprio sacerdote che viene designato con il termine knjez (Informazione orale del prof. H. Schuster-Šewc).

29 Cf. SETKA 1 I, 179, SKOK III, $51-52$ e $A R j$. s.v. pö́p e glagòljaš. 
dello slavo ecclesiastico prezviterŭ, prozviterŭ. I termini sono infatti ampiamente documentati nei testi glagolitici ${ }^{\mathbf{3 0}}$.

Si ricollega indirettamente al lat. presbyter anche il kajkavo jašprišt, jesprišt, derivante dall'aat. erzipriestar (attraverso la mediazione dell'ungherese esperest) $<$ lat. archipraesbyter, termine designante l'arciprete preposto a più parrocchie ${ }^{31}$.

Il grecismo presbyter, incomprensibile nel suo vero significato a chi non sapeva il greco, subì un incrocio con praebitor (da praebeo) che indicava quell'impiegato incaricato di dare vitto ed alloggio ai funzionari che si trovavano fuori sede per esigenze di servizio. Come il praebitor curava la salute ed il mantenimento materiale dei funzionari in viaggio, così il praebiter curava la salute dell'anima dei cristiani che si consideravano viaggiatori e pellegrini su questa terra ${ }^{32}$. A quest'ultimo termine si collega una voce diffusissima in territorio čakavo fin dal XIII secolo, ampiamente attestata nei testi glagolitici e due volte anche dalle epigrafi glagolitiche ${ }^{33}$ : prvad. Si tratta di un prestito dall'antico friulano prévidi (friul. mod. prédi) $<$ lat. praebiter $^{34}$.

Per completezza ci soffermeremo anche sulle denominazioni del sacerdote nell'esercizio di particolari attività, quali p. es. la celebrazione della messa, la confessione, l'educazione religiosa ecc. In questo genere di termini lo sloveno mášnik ed il croato mïsnik designanti letteralmente l'officiante la messa sono in uso fin dai tempi antichi. In sloveno questo termine è diffuso già in epoca protestante e registrato in tutti i dizionari più antichi indicando anche genericamente il sacerdote ${ }^{35}$. Il termine croato mìsnik è in uso già nel XV sec. ${ }^{36}$. Entrambe le voci si collegano con il termine che indica la messa, máša, rispettivamente mìsa, per l'origine del quale gli studiosi propendono piuttosto ad una derivazione diretta dal latino senza la mediazione del germanico ${ }^{37}$.

Somiglianze lessicali si registrano anche per la denominazione del confessore. Sia lo sloveno che il croato usano lo stesso termine spovedník, rispettivamente ispovjèdnik, di diffusione molto ampia nell'area degli slavi cattolici. Sarà da rilevare inoltre che il termine sloveno è attestato già nel manoscritto di Stična ${ }^{38}$. In croato registriamo però anche un prestito dalla voce italiana confessore, cioè konfêsur, che resta però limitato a sporadici autori di Ragusa del XVII sec., il che vale anche per il

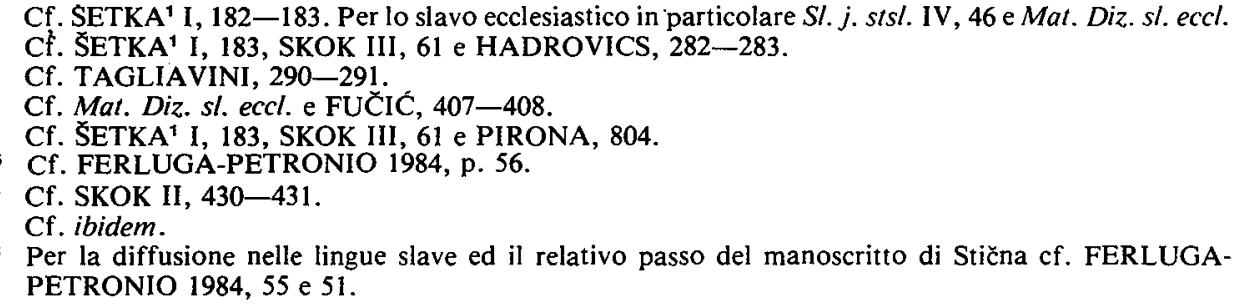


termine sačerdot ed il suo derivato sačerdocio nel significato rispettivamente di "sacerdote" e "sacerdozio"39.

Somiglianze si notano ancora per il termine che indica l'insegnante di religione, nello sloveno katehét, nel croato katèheta (secondo la pronuncia greca con la $h$ ) oppure la variante più rara katèketa (con la pronuncia $c h>k$ ), in Istria anche katihet, katiket. Mentre per lo sloveno non ci sono dubbi circa la derivazione diretta dal lat. eccl. catecheta, in croato il termine può essere penetrato sia atraverso il gr.

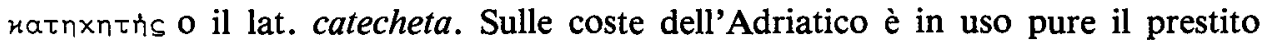
dall'italiano catechista, cioè katekista ${ }^{40}$.

Sulle coste della Dalmazia e dell'Istria sono inoltre diffuse delle abbreviazioni che si pongono dinanzi ai nomi dei sacerdoti, del tutto sconosciute nello sloveno. Così in Dalmazia si usa don già dal XVII sec. in poi (dun nelle Bocche di Cattaro), sotto l'influsso dell'italiano don (abbreviazione del lat. dominus). Esistono però ancora delle forme più antiche düm (XII sec.), döm (dal XIV al XVIII sec.) domin (XV e XVI sec.). E' tuttora viva la forma femminile dûmma (anche dûvna) nel significato di "monaca, badessa"441.

Fra le epigrafi glagolitiche, soprattutto in Istria, è ampiamente attestata l'abbreviazione pre (dall'it. prete) con la variante ikava pri (che costituisce però un hapax $)^{42}$.

Anche i termini che designano il parroco si presentano molto interessanti ad un'analisi comparativa tra le due lingue. Si osserva innanzitutto l'uso contemporaneo del medesimo termine, cioè dello slov. žúpnik e del cr. žüpnik, e l'influsso in questo caso del croato sullo sloveno. Lo șloveno žúpnik è registrato infatti molto tardi, appena nel Deutsch-slovenisches Taschenwörterbuch dello Janežič nel 1867. Fino ad allora si usava fin da epoche molto antiche fájmošter (anche nella forma farmošter dei protestanti) < mat. bav. *pharremeister significante "Pfarrer" ("parroco“). Il termine compare comunque già nel Manoscritto di Stična, particolarmente ricco di termini riferentisi alla gerarchia ecclesiastica. Il parroco veniva molto spesso designato semplicemente con fár, il termine più generico per sacerdote. La sostituzione con žúpnik va vista nell'ambito del processo di slavizzazione che cominciò a diffondersi in Slovenia negli anni Settanta del secolo scorso. la paura di un'eccessiva germanizzazione lessicale faceva introdurre termini da altre lingue slave: croato, russo, ceco. Questo fenomeno sostitutivo sta alla base - come avremo modo di vedere - anche di alcuni termini sloveni designanti i monaci ed i monasteri.

Il termine in questione è comunque molto antico e panslavo e si collega a žúpa, la più antica designazione slava per il distretto di una comunità, a capo della quale

\footnotetext{
Cf. SKOK II, 139 e III, 182.

Per le forme in questione si cf. SETKA ${ }^{1}$ I, 126 e SKOK II, 62.

4 Per le voci relative cf. SKOK I, 455-456, SETKA ${ }^{1}$ II, 47-48, ARj s.v.

42 Cf. FUČIC, 407 e SKOK III, 61.
} 
c'era lo žúpan. Ma nel corso del tempo il termine žúpa passò dall'amministrazione civile a quella ecclesiastica ad indicare la parrocchia presso i cattolici ${ }^{43}$.

Lo sloveno ed il croato hanno in comune ancora un termine designante il parroco, però in entrambe le lingue a livello dialettale. Inoltre in tutte e due le lingue si tratta di prestiti a seconda del territorio di diffusione, dall'ungherese o dal friulano. Così lo sloveno del Prekmurje conosce plevanuš/plivanuš $<$ dall'ungh. plébános $<$ lat. eccl. plebanus che era ufficialmente la designazione del sacerdote a capo di una plebs, una parrocchia di campagna (in contrapposizione alla parochia, parrocchia di città). Dall'ungherese trae origine anche il kajkavo plebanoš (XVI sec.) con numerose varianti, databili dal XV al XVII sec.: plebanus, plibanuš, prebanoš, prebanuš, pribanuš.

Dal friulano plavàn (ma anche plevàn, plovàn, cf. PIRONA, 780) trae origine plavan nella Val di Resia, ma anche tutta una serie di termini delle parlate čakave dell'Istria e della Dalmazia settentrionale: plòvan, plavan, plêvan, pelvan ${ }^{44}$.

Nel significato di parroco riscontriamo nel croato dialettale (Istria e Dalmezia) il termine kùrat che trae origine dall'italiano curato. Questa voce è presente anche nello sloveno kurát, designa però il cappellano di una comunità, specialmente se accompagnata da aggettivi quali p.es. bolniški, jetniškitis

Sempre nel litorale dalmata è registrata la voce parok (sconosciuta allo sloveno) che trae origine dal lat. parochus. I serbi usano a loro volta pàroh, derivante dal gr. $\pi \alpha \rho \circ \times O \varsigma$, da connettersi al verbo $\pi \alpha \rho \varepsilon \times \omega$ "somministrare, servire". In latino il grecismo parochus era l'equivalente del copiarius, dell'impiegato cioè incaricato di alloggiare e di fornire viveri per il viaggio dei funzionari statali. Anche questa è quindi una voce che passò dall'amministrazione civile a quella ecclesiastica ${ }^{46}$.

Ancora in croato una voce ormai desueta è stadbenik, di uso liturgico, che indica letteralmente il pastore, colui che guida lo städo "il gregge"447.

Per completezza citeremo per entrambe le lingue anche il termine dekàn, rispettivamente dèkan, derivanti entrambi dal lat. decanus con il quale si designa il vicarius foraneus, il sacerdote cioè che viene preposto dal vescovo a più parrocchie ${ }^{48}$.

$43 \mathrm{Cf}$. ARj sotto le rispettive voci. L'etimo di žúpa, žùpan è quanto mai controverso. Si è tentato di collegare le voci, ma senza successo, sia con lingue indoeuropee e non indoeuropee (SKOK III, 687).

44 Per gli etimi e le forme čakave cf. SKOK II, 689, per le forme kajkave HADROVICS, 416-417. Le forme dialettali slovene sono tratte da $\mathrm{Mat}$. SLA.

45 Cf. ARj s.v., SKOK II, 240-241 e SSKJ II, 532.

46 Cf. ARj s.v., SKOK II, 610 e TAGLIAVINI, 303-304.

47 Cf. ARj s.v. e SKOK III, 324.

48 Cf. SSKJ I, 335 e SETKA ${ }^{2}, 62$. 
Il quadro lessicale è assai meno vario per $i$ termini che designano $i$ gradi superiori della gerarchia ecclesiastica quali il canonico, il vescovo, il cardinale, il papa. Si tratta nella maggior parte dei casi di nomi tratti dalla terminologia ufficiale della Chiesa, direttamente dal latino oppure attraverso la mediazione germanica, in alcuni casi si nota però pure l'influsso del greco, specialmente nei testi glagolitici.

Dicesi canonico il sacerdote che viene scelto dal vescovo a far parte del capitolo sia della chiesa cattedrale o collegiale, al quale spetta di assolvere alle funzioni più solenni della chiesa. Il canonico, scelto dal vescovo a presiedere il capitolo, veniva chiamato anticamente, durante il medioevo, praepositus "preposto" (da cui l'italiano prevosto).

Per quanto riguarda il primo termine, cioè canonico, sia lo sloveno kanónik che il croato kànonik derivano direttamente dal lat. eccl. canonicus. In sloveno il termine compare dall'epoca del protestantesimo in poi, però anche fra $i$ protestanti è di uso raro. In croato la forma kànonik è attestata nel XV sec., riscontriamo però kànunik nel XIII, kanovnik dal XV al XVII sec. ${ }^{49}$

Per quanto concerne il secondo termine, cioè prevosto, sia lo sloveno che il croato conoscono la voce dotta prepòzit (PLET., 266) ovverossia preposit, prepozit, che trae origine direttamente dal lat. eccl. praepositus ${ }^{50}$. Entrambe le lingue conoscono comunque anche il termine che deriva da una variante del lat.praepositus, cioè propositus, ma non direttamente, bensì attraverso la mediazione del germanico. Si tratta dello slov. pròšt e del croato prošt, limitato quest'ultimo alle zone limitrofe del territorio etnico sloveno. La voce in questione trae origine dal mat. brobest, probest, probst (si cf. l'aat. prôbôst $<$ a. fr. provost $<$ lat. propositus) ${ }^{51}$.

Il croato conosce inoltre la voce prepošt, caratteristica dei dialetti kajkavi, che deriva dall'ungherse prépost ${ }^{52}$. Il čakavo conosce anche le forme prepušt, pripošt, pripušt attestate nel XVI sec..$^{53}$

Per ciò che riguarda le designazioni per il vescovo, esse traggono origine sia in sloveno che in croato, anche se attraverso per vie di mediazione diversa, dal lat. episco-

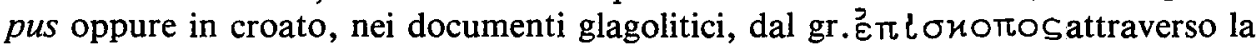
mediazione dello slavo ecclesiastico. L'є̌ $\iota \sigma$ น dai tempi antichissimi un funzionario dell'amministrazione civile, una specie di ispettore, sovrintendente. Soltanto nel II sec. incominciò ad essere usato per indicare il capo delle singole comunità cristiane. Il grecismo episcopus era molto raro nel latino classico ed appariva comunque nell'originario significato di "ispettore". An-

49 Cf. SKOK II, 35 e ARj s.v.

so Sarà da notare comunque che il termine sloveno viene registrato soltanto dai dizionari di Cigalè e Pleteršnik.

51 Si cf. STRIEDTER-TEMPS, 202 e SKOK III, 7.

52 Cf. SKOK, ibidem.

53 Cf. SKOK, ibidem e SETKA' II, 144-145. Nell'ARj si prospetta l'origine dall'it. preposto. 
che in questo caso notiamo il passaggio di un termine dall'amministrazione civile a quella ecclesiastica ${ }^{54}$.

Lo sloveno škòf è un prestito dall'aat. biscof ${ }^{55}$. Il Pleteršnik oltre la forma škòf (II, 632) cita anche la forma bîskup $(I, 27)$ precisando però trattarsi di un croatismo penetrato soprattutto nella lingua dei quotidiani sloveni. La presenza di questo particolare tipo di croatismo deve anch'essa ascriversi a quel fenomeno di slavizzazione lessicale diffusa in Slovenia negli anni Settanta del secolo scorso, fenomeno a cui si è già accennato a proposito del termine župnik.

A livello dialettale riscontriamo anche la voce bîškup nella Bela Krajina, chiaramente sotto l'influsso del kajkavo, però accanto alla forma škòf. Sempre a livello dialettale sono interessanti veškul/veškol (prestito dal friulano vèscul, PIRONA, 1270) della Val di Resia e püspek/püspäk del Prekmurje, prestito dall'ungherese püspö $k^{56}$.

Anche per il croato bìskup si era supposta dapprima l'origine germanica dall'aat. biscof $f^{57}$. Lo Skok ${ }^{58}$ propende invece per una derivazione dal lat. episcopus, poichè ad una $o$ dell'antico alto tedesco non può corrispondere una $u$ slava, d'altra parte ad una $o$ stretta romanza corrisponde regolarmente una $u$ nello slavo. La $e$ iniziale sarebbe caduta, come accade a tutti i grecismi della latinità dalmata. La $b$ iniziale sarebbe dovuta in ultima anlisi alla dissimilazione di $p-p$ di episcopus.

Sarà da notare inoltre nei documenti e nelle epigrafi glagolitiche la forma

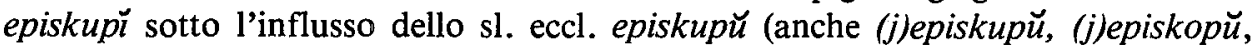
mentre gli ortodossi usano ancora i termini jepiskop, jepiskup ${ }^{59}$.

Saranno da registrare inoltre ancora due termini che appaiono esclusivamente in croato. 11 primo, eršeg, eršek, prestito dall'ungherese érsek, è limitato al territorio kajkavo $^{60}$. Il secondo, stadničar, derivato da stàdo "gregge", è ormai desueto ${ }^{61}$.

Per completezza citeremo una voce comune ad entrambe le lingue, lo slov. prelát ed il cr. prèlat, dei trasparenti latinismi dal lat. praelatus, designanti genericamente un alto dignitario ecclesiastico ${ }^{62}$.

\footnotetext{
Cf. Encicl. Catt. XII, c. 1312.

Cf. STRIEDTER-TEMPS, 219.

Le informazioni riguardanti le espressioni dialettali sono tratte da Mat. SLA.

47 Cf. MIKL., Christ. term., 13.

58 Cf. SKOK I, 157.

59 Cf. Mat. Diz. sl. eccl. e ARj s.v.

60 Cf. FINKA I, 3, 542. L'etimo di érsek non è stato ancora ben chiarito, ma si propende per la derivazione dall'antico francese archevêque (HADROVICS, 215).

61 Si v. anche la già menzionata voce stadbenik "parroco".

62 Cf. SSKJ III, 1049 e ARj s.v.
} 
Molto semplici sono dal punto di vista etimologico i termini designanti il cardinale, slov. kardinál e cr. kardinal voci dotte dal lat. cardinalis $^{63}$. Per lo sloveno è da menzionare nel dizionario del Gutsmann accanto all'usuale kardinal anche erdezhjak, che potrebbe essere tradotto "porporato" (da connettersi all'aggettivo raèc "rosso"). A sua volta anche il croato conosce un termine originale tutt'ora in uso: il calco stöžernik. Stöžer significa infatti "cardine" ${ }^{\text {"64 }}$.

Non sono, ovviamente, numerose nelle due lingue le denominazioni per il papa e denotano per ovvi motivi perecchie similitudini. Lo slavo ecclesiastico conosceva due termini per indicare il papa: papež e $p a p a^{65}$. Il primo fu introdotto in Moravia e nel territorio delle Alpi slovene dai missionari di Regensburg e di Salisburgo prima della missione dei SS. Cirillo e Metodio nella forma aat. babes, pabes, e si diffuse tra le lingue degli Slavi di confessione cattolica, fra cui anche nello slov. pápež. Il se-

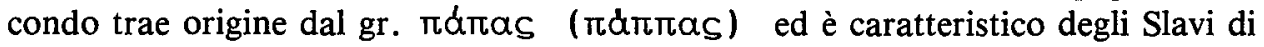
confessione ortodossa ${ }^{66}$. Presso i croati viene usato il secondo termine, pâpa, che trae origine direttamente dal lat. papa e viene a volte allargato anche in rîmski-pâpa, rim-pâpa, quest'ultimo dall'espressione turca dispregiativa rimpapasi ${ }^{67}$.

Entrambe le lingue conoscono l'espressione "santo padre", in slov. svéti ôče, in cr. svêti òtac, in entrambe le lingue viene usato il termine apostólik, rispettivamente apostòlik $k^{68}$, quest'ultimo è attestato anche nei testi glagolitici già dal XIV sec., però nella forma apustoliǩ (con metatesi da apostul $<$ mlat. apostulus) ${ }^{69}$.

Soltanto nei testi glagolitici troviamo nel significato di papa il termine arhijerej $7^{70}$, dovuto all'influsso dello slavo ecclesiastico. Questa voce è caduta oggi completamente in disuso presso i croati ed è viva soltanto nella chiesa ortodossa.

Molto interessante e varia si presenta la terminologia che riguarda i monaci ed i monasteri, soprattutto per l'influsso di alcuni termini croati sullo sloveno, ma anche per una ricca presenza di voci nella letteratura glagolitica e parecchi prestiti dall'italiano sulla costa dalmata.

In sloveno il termine più antico e più usato dal Cinquecento in poi per designare il monaco è menì che in croato è relegato solo ai testi glagolitici sotto l'influsso dello slavo ecclesiastico münihü nella forma mnih̆ con la perdita di $\breve{u}$ (si cf. anche il

63 Il croato antico conosce anche kardinao (dal XVI sec. in poi, kardino (caratteristico di Ragusa) e le forme čakave gardinal, gardinao (dal XV al XVIII sec.), gardènao (nel XVI e XVII sec.) (cf. ARj s.v.).

64 Cf. ARj s.v. e SKOK II, 50

65 Cf. Sl. j. sisl. III, $13-14$.

66 Cf. STRIEDTER-TEMPS, 189 e KIPARSKY, 155. Per quanto riguarda la diffusione nelle lingue degli slavi cattolici e degli slavi ortodossi cf. FERLUGA-PETRONIO 1984, 86 e 87.

67 Cf. SKOK III, 8-9 e SETKA $1,164$.

68 Cf. SSKJ, 57 e J. Jurančič, Srbskohrvatsko-slovenski slovar, Ljubljana 1968, 18.

69 Cf. Mat. Diz. sl. eccl. e ŠTKA' I, 48.

70 Anche questo termine è tratto da Mat. Diz. sl. eccl. 
corrispettivo femminile mniha) e quella più rara manih $\breve{i}$ con il passaggio di $\breve{u}>a^{71}$. Lo sl. eccl. munihu è prestito dall'aat. munich. Si tratta di un termine introdotto in terra morava dai missionari germanici e qui recepito dalla missione slava dei SS. Cirillo e Metodio. Ebbe vasta diffusione fra gli slavi di confessione cattolica ${ }^{72}$. Gli slavi ortodossi usano invece prestiti dal gr. $\mu$ ov $\alpha \times \delta_{S}$, , per cui nell'antico serbo è riscontrabile monah (fem. monahija, monahinja). L'antico croato monak è invece tratto o dal lat. monachus o dall'it. monaco, mentre il fem. monaca trae origine dall'it. monaca ${ }^{73}$.

Accanto a menì in sloveno è attestato dal dizionario dello Cigalè in poi (cioè dalla seconda metà dell'Ottocento) il termine redóvnik, il quale a somiglianza del già menzionato žúpnik può considerarsi un'innovazione lessicale sotto l'influsso del croato. Redòvnik in croato con il corrispondente redòvnica è il termine più usuale per indicare il monaco. Si tratta di una voce di diffusione panslava da connettersi a rêd "ordine". In croato la troviamo attestata già nei testi glagolitici del XIV sec., i quali conoscono anche altri termini per designare i monaci, ma redòvnik è l'unico che sopravvive nella lingua odierna ${ }^{74}$.

Così l'antico croato conosceva crnorízac (e crnorizica), che compare però raramente nei testi glagolitici del XVI sec. sotto l'influsso dello slavo ecclesiastico črŭnoriž́c̆, ed è caratteristica soprattutto dei documenti anticoserbi ${ }^{75}$. Quest'espressione indica "colui che è vestito di nero": $c \hat{n} n$ "nero" e rìza (anticamente) $=$ "veste".

Nel litorale dalmata erano diffusi inoltre kòludar, kòludrica sotto l'influsso dello slavo ecclesiastico che conosce appunto kalugerŭ (kalugerica), prestito dal

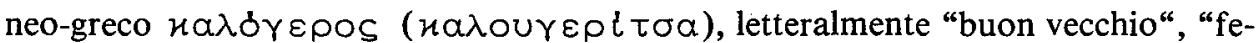
lice nella vecchiaia". Le forme più diffuse nel serbocroato sono comunque kàluđer, kàluđerica, nell'antico serbo kalođer, kalođerica. Nella voce dalmata kòludar il passaggio $a>o$ attesta la sua particolare antichità, mentre in tutte le voci in questione il passaggio del gruppo $g r>d r$ rispetto allo slavo ecclesiastico è dovuto probabilmente a dissimilazione di $k-g>k-d^{6}$.

Per ciò che riguarda il termine designante il convento sia lo sloveno che il croato usano oggigiorno lo stesso termine samostán, rispettivamente sàmostan ${ }^{77}$. La voce slovena in questione è comunque registrata appena dallo Cigalè in poi. Si tratta anche in questo caso, come abbiamo già osservato precedentemente per redóvnik, di un termine introdotto sotto l'influsso del croato. Anticamente era diffuso in sloveno

71 Cf. Mat. Diz. sl. eccl. e SKOK II, 453.

72 Per l'etimo si cf. KIPARSKY, 152, per la diffusione in territorio slavo invece BERNEKER II, 75.

73 Si cf. BERNEKER ibidem, SKOK ibidem, e SETKA ${ }^{1}$ I, 156-157. Per la designazione della monaca lo sloveno usa invece il termine núna $<$ aat. nunna (Cf. BEZLAJ II, 230).

74 Cf. ARj s.v. e Mat. Diz. sl. eccl.

75 Cf. ARj, Mat. Diz. eccl. e MIKL., Lex., 1122.

76 Cf. Mat. Diz. sl. eccl.; Sl. j. stsl. II, 8; SETKA ${ }^{1}$ I, 120-121 e SKOK II, 129.

77 Cf. SSKJ e ARj s.v. 
dal Cinquecento in poi il termine klóšter che era caratteristico - nella forma klòštar - anche nelle regioni nordoccidentali della Croazia, non solo fra i dialetti kajkavi e čakavi, ma bensi anche fra quelli štokavi. Questa antica voce, comune ad entrambe le lingue, trae origine dall'aat. klôstar < lat. claustrum. Sarà interessante notare che a Ragusa sono attestati dal XVI al XVIII sec. latinismi dotti kläustro, kläuštro, kläustar che traggono origine direttamente da claustrum $^{78}$. E' infine nuovamente caratteristico di Ragusa e tuttora ricorrente il prestito dall'it. convento: kònven(a)t, anticamente ance kumven(a)t, kunven $(a) t^{79}$.

Il croato è particolarmente ricco di denominazioni per il convento che sono sconosciute allo sloveno. E' il caso di tutta una serie di voci che trae origine dallo slavo

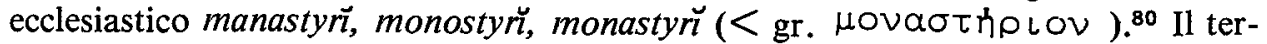
mine si propagò sia fra i serbi che fra $\mathrm{i}$ croati. La forma più usata era mànastir, $\mathrm{a}$ Ragusa è attestata a volte la forma nämastir, con la metatesi $n-m$. Esclusivamente nei manoscritti e libri croati dal XIII al XVI sec. è attestato molstir (da una forma del latino volgare non documentata monisterium, con la dissimilazione $m-n>m$ $-l$ ). Nei dialetti čakavi sono inoltre riscontrabili anche le forme mojstir e mostir ${ }^{\mathbf{B 1}}$.

Per indicare l'abbate sia lo sloveno che il croato adoperano lo stesso termine opát rispettivamente òpat. Si tratta di termini molto antichi, in croato è attestato addirittura nella Baščanska ploča, in sloveno è registrato fin dal dizionario del Megiser in poi. L'origine di tale termine che è diffuso pure nelle lingue slave occidentali, non è del tutto chiarita. Si propone la derivazione dall'aat. *appat, ma non senza difficoltà, poichè nell'antico alto tedesco esiste soltanto la forma $a b b a t^{82}$. In croato accanto ai derivati òpatica "badessa" e opàtija "abbazia" sono da riscontrare alcune forme molto rare derivanti o dal latino o dall'italiano, quali àbat, oppure dal latino abatiša $\left(<\right.$ abbatissa) oppure dall'italiano (a)badesa e abacija ${ }^{83}$.

Per designare il superiore del convento entrambe le lingue usano il latinismo prior, rispettivamente prìor, (lo sloveno anche il calco prédnik, fem. prédnica), come pure entrambe le lingue conoscono il latinismo páter con il quale si indica il monaco che è stato ordinato sacerdote ${ }^{84}$. I monaci usano chiamarsi fra loro con termini quali "fratello"e "sorella" (brät e sêstra/sèstra $)^{\beta 5}$. Sempre al concetto di fratello è legato il latinismo frâtar con cui viene designato il frate appartenente ai vari ordini. Frâtar con la rispettiva abbreviazione frà (derivante dall'italiano frate) preposta al nome del monaco, è diffuso dal XIV sec. in poi a Ragusa, nella Dalmazia settentrionale nonchè in Bosnia ed Erzegovina ${ }^{86}$.

\footnotetext{
Cf. STRIEDTER-TEMPS, 151; BEZLAJ II, 47; ARj s.v. e SKOK II, 93.

Cf. ARj s.v. kònvenat; SETKA ${ }^{1}$ II, 93 e 100; SKOK III, 142.

Cf. Sl. j. stsl. II, 186-288.

1 Cf. per le rispettive voci e gli etimi SETKA ${ }^{1}$ I, 148 -149, Mat. e SKOK II, 453-454.

2 Cf. BERNEKER I, 22 e BEZLAJ II, 250.

$3 \mathrm{Si}$ cf. per queste voci ARj e SKOK III, 560.

4 Cf. ARj s.v. e SSKJ III, 1003 e III, 560.

Cf. SETKA ${ }^{i}$ III, 3 e $200-201$.

6 Cf. ARj s.v. e SKOK I, 529.
} 
Come abbiamo già accennato all'inizio di questo articolo i termini per la gerarchia ecclesiastica in sloveno ed in croato presentano molti punti in comune, soprattutto per ciò che riguarda i prestiti dal latino ecclesiastico che sono più numerosi ed investono particolarmente la sfera delle voci dotte appartenenti al linguaggio liturgico quali il clero, il chierico, il diacono, il decano, il canonico, la voce dotta per prevosto (prepòzit/prepozit), il cardinale, il prelato. Ma già fra questi termini notiamo per il croato delle varianti fonetiche che denotano oltre che la recezione dal latino anche un'altra dal greco attraverso lo slavo ecclesiastico dei testi glagolitici. Possiamo così notare accanto alle forme klêr, klêrik anche klîr, klìrik oppure accanto a prezbiter anche prezviter, prozviter.

In croato le voci sotto l'influsso dello slavo ecclesiastico sono comunque le più importanti dopo i prestiti dal latino e sono testimoni del forte legame esistente con la Chiesa Orientale. Si tratta per la maggior parte di nomi designanti gli alti gradi della gerarchia ecclesiastica oppure monaci e monasteri (jerej, arhijerej, svetitelj, episkupü, crnorizac, kòludar, kàluđer, mänastir ecc.). Questo tipo di termini, fra i quali ci sono moltissimi grecismi, praticamente non esiste nello sloveno ad eccezione di jerej usato una sola volta da Krelj, da attribuire quindi alla profonda erudizione di questo riformatore sloveno. Lo stesso termine nelle forme iêro/ệro appare come una specie di relitto dialettale (finora non sufficientemente spiegato) nella Val di Resia.

In questo particolare settore lessicale il croato si differenzia dallo sloveno per tutta una serie di romanismi o meglio italianismi, molti dei quali sono tuttora diffusi in Istria, lungo tutta la costa dalmata e nelle isole, testimoni dei legami storici con la Repubblica di Venezia. Si tratta soprattutto di termini che designano il sacerdote nell'espletamento delle sue varie funzioni (predikator, konfèsur, sačerdot, ecc.), il parroco (plavan nelle sue numerose varianti), i monaci ed il convento (monaka, abat, (a)badesa, abacija, konven(a)t ecc.) e tutta una serie di abbreviazioni reverenziali che si antepongono ai nomi dei sacerdoti e dei frati (don, dun, düm ecc., pre, pri, frà ecc.). Queste voci sono completamente sconosciute allo sloveno che annovera fra i romanismi soltanto due prestiti dal friulano: plavan nella Val di Resia (è questo anche il suo unico esempio in comune con il croato) e vêškul/vêškol, sempre nella Val di Resia. La completa assenza di italianismi nello sloveno in questo particolare settore lessicale è indice di un processo storico e perciò anche lessicale diverso dal croato, maggiormente legato al mondo germanico.

Ciò si evince anche dall'analisi comparativa dell'influsso del germanico su questo tipo di termini. Si può osservare infatti che i prestiti in tal senso sono più numerosi in sloveno che in croato ed investono un po' tutto il campo semantico in questione, dai gradi superiori del clero (pápě̌, škòf, pròšt, opát), al semplice sacerdote (fár, fájmošter, prídigar ecc.), ai monaci e monasteri (menìh, núna, klóšter), alla designazione stessa per la Chiesa (cérkev). Alcuni di questi sono comuni anche al croato (p. es. cr̂kva, klôštar, òpat anticamente mnih̆ /maniȟ̆), dobbiamo però precisare che per molte di queste voci in croato l'origine dal germanico non è del tutto sicura e 
vengono perciò proposte anche ipotesi di derivazione dal greco (p. es. crkvva) oppure dalla latinità balcanica (p. es. biskup).

Possiamo osservare inoltre che molti di questi termini, caratteristici soltanto per lo sloveno (come p. es. pápež) o ad entrambe le lingue (p. es. menìh/mnihĭ), sono dei moravismi, cioè introdotti in terra morava dai missionari germanici prima dell'arrivo dei fratelli di Salonicco, recepiti quindi da Cirillo e Metodio e diffusi soprattutto fra gli slavi cattolici. Si tratta, quindi, di antichi prestiti dal germanico nella lingua di Cirillo e Metodio, testimonianze del sostrato linguistico-culturale della Chiesa occidentale in terra morava.

L'apporto, infine, dell'ungherese in questo particolare campo semantico è minimo in entrambe le lingue, anche se notiamo qualche termine in più per il croato, dovuto principalmente a cause di confinanza geografica. Così p. es. nel dialetto di Prekmurje registriamo plevanuš/plivanuš e nej kajkavo plebanoš. In ogni caso l'incidenza dall'ungherese si può definire del tutto marginale.

Da non sottovalutare invece i termini formatisi nell'ambito linguistico slavo che risultano più numerosi nel croato e riguardano soprattutto i nomi designanti il secerdote nello svolgimento di varie funzioni (p. es. svècenik, dùhovnik, glagòljaš, ispovjèdnik, ecc.). Da notare anche due calchi per la designazione del cardinale e del convento: stöžernik e sămostan. Nulla di strano perciò che lo sloveno si sia avvalso in epoche più recenti, quando il processo di slavizzazione s'era fatto più sentito, anche di croatismi. E' ben vero che l'antico fár viene sostituito con duhóvnik, con un termine proprio che in varie forme e significati troviamo attestato dal Cinquecento in poi, ma si operano pure delle sostituzioni con termini desunti dal croato: fájmošter con žúpnik, klóšter con samostán, accanto all'antico menì comincia ad apparire redóvnik.

Possiamo comunque notare anche qualche influenza dello sloveno sul kajkavo, dovuta a cause di vicinanza geografica (p. es. il kajkavo káplan nel significato di "vice-parroco" sotto l'influsso dello slov. kaplán).

L'analisi comparativa fra le due lingue in questo particolare settore della terminologia religiosa si presenta indubbiamente interessante non solo per capire le similitudini e le differenze semantiche, la minore o maggiore diffusione di certi termini (anche con significati diversi) nell'una e nell'altra lingua, e le loro reciproche influenze.

Essa si rivela particolarmente interessante per cogliere meglio sia l'origine sia le vie di mediazione attraverso le quali un determinato termine si è diffuso in territorio linguistico sloveno o croato oppure in ambedue. 


\section{ABBREVIAZIONI BIBLIOGRAFICHE}

$\operatorname{ARj}$

Rječnik hrvatskoga ili srpskoga jezika, JAZU, Zagreb 1880-1975.

BERNEKER

BEZLAJ

\section{BRÜCKNER}

CIGALE

Encicl.Catt.
E. BERNEKER, Slavisches etymologisches Wörterbuch, I-II, Heidelberg 1908-1913.

F. BEZLAJ, Etimološki slovar slovenskega jezika, I, II-, Ljubljana 1976, 1982-.

A. BRÜCKNER, Stownik etymologiczny jezyka polskiego, Kraków 1927.

M. CIGALE, Deutsch-slovenisches Wörterbuch, I-II, Ljubljana 1860.

Enciclopedia Cattolica, Città del Vaticano, $1948-1954$.

FERLUGA-PETRONIO 1984 F. FERLUGA-PETRONIO, La Chiesa in Slovenia, Analisi filologico-etimologica della gerarchia ecclesiastica con particolare riguardo ai testi del Cinquecento, Trieste 1984.

FERLUGA-PETRONIO 1990 F. FERLUGA-PETRONIO, I nomi della gerarchia ecclesiastica in croato, Studia Patavina 37 (1990) I, Padova 1990, p. 97-117.

FINKA

B. FINKA, Rječnik hrvatskoga kajkavskoga književnog jezika, JAZU, Zagregb 1984-.

FUČIĆ

GUTSMANN

HADROVICS

HRASTE-ŠIMUNOVIĆ

JANEŽIČ

KARADŽIĆC

KIPARSKY

KLUGE-MITZKA

Mat.Diz.Prot.
B. FUČIĆ, Glagoljski natpisi, JAZU, Zagreb 1982.

O. GUTSMANN, Deutsch-windisches Wörterbuch mit einer Sammlung der verdeutschen windischen Stammwörter, Klagenfurt 1789.

L. HADROVICS, Ungarische Elemente im Serbokroatischen, Köln-Wien, 1985.

M. HRASTE-P. S̆IMUNOVIĆ, Čakavischdeutsches Lexicon (Unter Miterbeit und Redaktion von Reinhold Olesch), Köln-Wien I (1979) - II (1981).

A. JANEŽIČ, Slovenisch-deutsches Handwörterbuch, Klagenfurt 1893.

VUK ST. KARADŽIĆ, Srpski rječnik, Nolit, Beograd 1975 (riproduzione della II ediz., Vienna 1852). V. KIPARSKY, Die gemeinslawischen Lehnwörter aus dem Germanischen, Helsinki 1934.

F. KLUGE-A. GÖTZE-A. SCHIRMER-W. MITZKA, Etymologisches Wörterbuch der deutschen Sprache, Berlin $1957^{17}$.

Materiale manoscritto per lo Slovar slovenskih protestantskih piscev, ZRC-SAZU, Ljubljana. 
Mat. SLA

Mat.Diz.sl.eccl.

MIKL., Christ.Term.

MIKL., Lex.

PIRONA

PLET.

POHLIN

\section{SCHNEEWEIS}

SKOK

Sl.j.stsl.

SP

SSKJ

STRIEDTER-TEMPS

STRIEDTER-TEMPS, Sbcr.

ŠTKA $^{1}$

ŠETKA ${ }^{2}$

TAGLIAVINI
Materiale manoscritto per lo SLA (= Slovenski lingvistični atlas), ZRS-SAZU, Ljubljana.

Materiale manoscritto per il Rječnik crkvenoslavenskoga jezika hrvatske redakcije, Staroslavenski zavod "Svetozar Ritig", Zagreb.

F. MIKLOSICH, Die christliche Terminologie der slavischen Sprachen. Wien 1875.

F. MIKLOSICH, Lexicon palaeoslovenico-graecolatinum, Vindobonae 1862-1865.

G.A. PIRONA-E. CARLETTI-G.B. CORGNALI, Il nuovo Pirona. Vocabolario friulano, Udine 1935. M. PLETERŠNIK, Slovensko-nemški slovar (I-II), Ljubljana 1894-1895.

M. POHLIN, Tu malu besedishe treh jezikov 1781, Faksimile der ersten Ausgabe, München 1973.

Die deutschen Lehnwörter im serbokroatischen in kulturgeschichtlichen Sicht, Berlin 1960.

P. SKOK, Etimologijski rječnik hrvatskoga ili srpskoga jezika, I-IV, Zagreb 1971-1874.

Slovnik jazyka staroslovenského (Lexicon linguae palaeoslovenicae) red. J.KURZ, Praha 1958-.

Slovenski pravopis, Ljubljana 1962.

Slovar slovenskega knjižnega jezika, Ljubljana 1970-.

H. STRIEDTER-TEMPS, Deutsche Lehnwörter im Slovenischen, Berlin 1963.

H. STRIEDTER-TEMPS, Deutsche Lehnwörter im Serbokroatischen, Wiesbaden 1958.

Dr.O.L. ŠETKA, Hrvatska kršćanska terminologija:

I Hrvatski kršćanski termini grčkoga porijekla, Sibenik 1940.

II Hrvatski kršćanski termini latinskoga porijekla, Makarska 1964.

III Hrvatski kršćanski termini slavenskoga porijekla, Makarska 1965.

Dr.O.L. ŠETKA, Hrvatska kršćanska terminologija, Split 1976.

C. TAGLIAVINI, Storia di parole pagane e cristiane attraverso $i$ tempi, Brescia 1963. 


\section{PRIMERJALNA ANALIZA IMEN ZA DUHOVŠČINO V SLOVENŠČINI IN HRVAŠČINI}

Primerjalna analiza med slovenskimi in hrvaškimi imeni za duhovščino ni zanimiva samo za spoznavanje njihove medsebojne podobnosti in različnosti ter njunih medsebojnih vplivov, temveč predvsem za boljše razumevanje izvora posameznih izrazov in jezikovnih poti, preko katerih so se zakoreninili in razŠirili na posameznih etničnih področjih. Iz primerjave med slovenskim in hrvaškim besednim gradivom ugotovimo, da je bil v tovrstnem besedišcu vpliv hrvaščine na slovenščino - in sicer v omejeni obliki večji kot obratni vpliv, predvsem v mlajših obdobjih. Glede izvora samega besedišča imata oba jezika veliko skupnih izposojenk iz cerkvene latinščine, hrvaščina se odlikuje po velikem številu izposojenk iz gršcine, predvsem preko cerkvene slovanšcine. Tovrstnega vpliva sploh ni opaziti v slovenščini, ki je pa po drugi strani pod večjim vplivom germanskih cerkvenih izrazov kot hrvaščina. Velik delež pri oblikovanju tovrstnega hrvaškega izrazja je imel romanski besedni zaklad, predvsem italijanščina. Tudi ta vpliv je v slovenščini takorekoč neopazen. Iz madžarščne sta oba jezika sprejela le nekaj besed, tako da je s te strani vpliv na imena za duhovščino le obroben. 